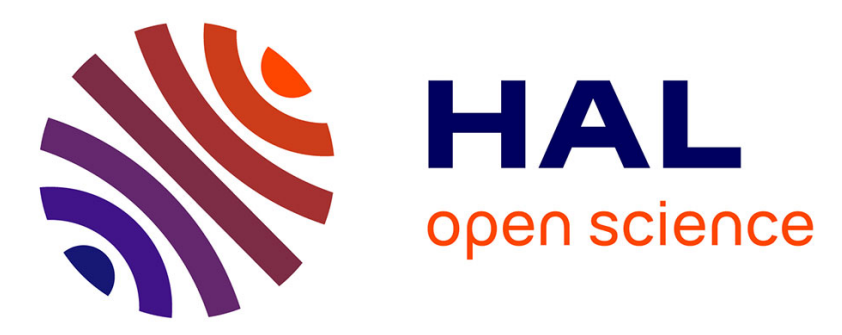

\title{
Realization of a Magnet Using an Antiferromagnetic Phase of Single-Chain Magnets
}

C. Coulon, Hitoshi Miyasaka, Wolfgang Wernsdorfer, Thierry Colin, Rodolphe Clérac

\section{- To cite this version:}

C. Coulon, Hitoshi Miyasaka, Wolfgang Wernsdorfer, Thierry Colin, Rodolphe Clérac. Realization of a Magnet Using an Antiferromagnetic Phase of Single-Chain Magnets. Physical Review Letters, 2009, 102, pp.167204. 10.1103/PhysRevLett.102.167204 . inria-00443849

\section{HAL Id: inria-00443849 \\ https://hal.inria.fr/inria-00443849}

Submitted on 12 Mar 2013

HAL is a multi-disciplinary open access archive for the deposit and dissemination of scientific research documents, whether they are published or not. The documents may come from teaching and research institutions in France or abroad, or from public or private research centers.
L'archive ouverte pluridisciplinaire HAL, est destinée au dépôt et à la diffusion de documents scientifiques de niveau recherche, publiés ou non, émanant des établissements d'enseignement et de recherche français ou étrangers, des laboratoires publics ou privés. 


\title{
Realization of a Magnet Using an Antiferromagnetic
}

\section{Phase of Single Chain Magnets}

\author{
Claude Coulon, ${ }^{1,2, *}$ Hitoshi Miyasaka, ${ }^{3}$ Wolfgang Wernsdorfer, ${ }^{4}$ \\ Thierry Colin, ${ }^{5}$ and Rodolphe Clérac ${ }^{1,2, *}$
}

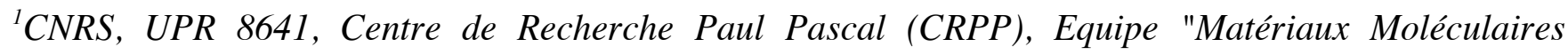
Magnétiques", 115 avenue du Dr. Albert Schweitzer, Pessac, F-33600, France.

${ }^{2}$ Université de Bordeaux, UPR 8641, Pessac, F-33600, France.

${ }^{3}$ Department of Chemistry, Graduate School of Science, Tohoku University, 6-3 Aramaki-Aza-Aoba, Aoba-ku, Sendai, Miyagi 980-8578, Japan.

${ }^{4}$ Institut Néel, CNRS \& Université Joseph Fourier, BP 166, 25 Avenue des Martyrs, 38042 Grenoble Cedex 9, France.

${ }^{5}$ Université de Bordeaux, IMB UMR CNRS 5251 and INRIA Bordeaux-Sud-Ouest EPI MC2, 351 Cours de la Libération, 33400 Talence, France.

\footnotetext{
*Towhom correspondence should be addressed.E-mail:coulon@crpp-bordeaux.cnrs.fr,clerac@crppbordeaux.cnrs.fr
} 
Abstract: A system presenting an antiferromagnetic (AF) phase of Single-Chain Magnets (SCMs) is studied at low temperatures. The phase diagram is first discussed to specify the magnitude of the interchain couplings. Then, we show experimentally and theoretically that slow relaxation of the magnetization can still be observed in the AF phase, with a maximum of the relaxation time close to the AF-paramagnetic phase transition. This counter intuitive result shows that materials presenting such an ordered state can be used to design high temperature magnets using SCM-based materials.

PACS numbers : 75.50.Xx, 75.10.Pq, 75.40.Gb, 75.75.+a, 75.30.-m

The design and optimization of nano-scale devices able to store information are two key issues affecting technology applications in the near future. As far as magnetic recording is concerned, information storage can be realized when the magnetization relaxation is slow enough to allow the freezing of a metastable magnetized state in absence of applied magnetic field. Since the pioneering work of R. J. Glauber in the 1960's (1), it has been established that slow magnetic dynamics can be obtained with chains combining the effects of a large magnetic anisotropy and ferromagnetic exchange interactions between the magnetic chain units (2). Although magnetically isolated chains do not order at a finite temperature, large short-range correlations along the chain are present and promote the appearance of slow magnetic relaxation. Surprisingly, the first experimental one-dimensional systems displaying slow magnetic dynamics were only reported in 2001 by D. Gatteschi and coworkers (3). Since this breakthrough, a relatively large number of new experimental systems have been described $(2,4-6)$ and the term Single-Chain Magnets (SCM) (4) has since been adopted for this new class of nano-magnets. Although SCMs are based on coordination compounds (transition metal ions and ligands), other systems incorporating metal atoms assembled on non magnetic substrates may also exhibit a similar dynamic behavior (7). Slow relaxation in SCMs is intrinsically a one-dimensional mechanism and by increasing the intrachain exchange interactions, high temperature SCMs could be theoretically obtained. In practice, a bulk material is composed of many chains and small interchain interactions (in most cases 
antiferromagnetic in nature) are irrevocably present. Consequently, three-dimensional ordering (typically an antiferromagnetic phase) is expected to be stable at low temperature and it is quite generally believed that slow relaxation of the magnetization no longer exists in this ordered phase (8). In this letter, we present experimental and theoretical results that unambiguously show the occurrence of slow relaxation of the magnetization in an antiferromagnetic phase of SCMs. A simple model, treating the interchain couplings in the mean field approximation, is developed to describe the phase diagram and the field dependence of the relaxation time. Hence, in contradiction to previous claims (8), the magnet property is preserved and furthermore enhanced, when a three dimensional antiferromagnetic order is present. This provocative result is an important step towards the design of high temperature magnets based on SCMs.

The material studied in this work is $\left[\mathrm{Mn}_{2}(5-\mathrm{MeOsaltmen})_{2} \mathrm{Ni}(\text { pao })_{2}(\right.$ phen $\left.)\right]\left(\mathrm{PF}_{6}\right)_{2}, \mathbf{1}$, where 5MeOsaltmen, pao, and phen are the $N$, $N^{\prime}-(1,1,2,2$-tetramethylethylene $)$ bis(5methoxysalicylideneiminate), pyridine-2-aldoximate and 1,10-phenanthroline ligands respectively. This sample has a one-dimensional structure (9). Its chains are made of $\mathrm{Mn}_{2} \mathrm{Ni}$ trinuclear units that can be considered effectively as $S=3$ spins at low temperature $(2,4,10)$. Compounds of the same family have already been published and described as SCMs down to very low temperature (4). The field dependence of their relaxation time has also been described and a maximum is observed when the applied field vanishes as predicted by the theory (10). To characterize the magnetic properties of $\mathbf{1}$, susceptibility measurements were first performed on polycrystalline samples as shown in inset of Fig. 1b. As for other SCM systems, a thermally activated regime is observed when plotting $\chi T$ versus $1 / T$ revealing the exponential dependence of the Ising-like intrachain correlation length (2). The corresponding activation energy gives an estimation of the intrachain exchange energy as $\Delta_{\xi}=4 J_{/ /} S^{2}$, with $J_{/ /}$being the intrachain magnetic interaction (11). We obtain: $\Delta / k_{\mathrm{B}} \approx 18 \mathrm{~K}$. While the $\chi T$ product saturates in other systems at low temperature due to finite size effects (12), here a marked decrease is observed at zero field in 
concomitance with a strong field dependence of $\chi T$. Neither effect can be explained using an onedimensional description and supports the existence of an antiferromagnetic ordered phase. Single crystal experiments were also performed using a homemade micro-SQUID apparatus by applying the field along the easy axis (13). Fig. 1a presents typical results obtained at low temperature scanning the field at a constant rate. Hysteresis loops are observed emphasizing that the material behaves as a magnet. Typically above $2.5 \mathrm{~K}$ it becomes possible experimentally to obtain reversible data (i.e. no hysteresis effects) sweeping the field slowly. An inflection point is found close to $40 \mathrm{mT}$ at $2.9 \mathrm{~K}$ for the $M$ vs $H$ data (i.e. a maximum of the susceptibility), but similar results are also obtained at higher temperature up to a maximum of $5 \mathrm{~K}$. The derivative of the magnetization was used to deduce the magnetic susceptibility as illustrated for a typical result shown in Fig. 1b. It is worth noting that data from powder and single crystal measurements are in agreement and both have been used to locate the susceptibility maximum as a function of temperature and applied field (14). This maximum is reported in Fig. 2 and provides a good approximation for the location of the phase transition between the paramagnetic and antiferromagnetic (AF) ground states. Both the temperature dependence of the zero field susceptibility and the shape of the deduced transition line are consistent with three-dimensional antiferromagnetic ordering. To confirm this analysis, a simple two-sublattice model has been developed to obtain the phase diagram in the Ising limit, treating the interchain couplings in the mean field approximation. To be general, exchange energies between opposite sublattices $\left(J_{\perp}\right)$ and between sublattices of the same kind $\left(J_{\perp}^{\prime}\right)$ should be considered (see Supplementary Information). Although such a simplified treatment cannot account for all experimental details, we were able to reproduce the main characteristics of the observed phase diagram. Considering the data obtained from both static and dynamic measurements (discussed below), the theoretical phase diagram has been optimized in order to simulate the experimental data (15). The calculated transition line given in Fig. 2 is consistent with experimental results. The field dependence of the magnetization of each sublattice $\left(m_{\mathrm{i}}\right)$ can also been calculated. As $m_{1}$ and $m_{2}$ have opposite values at zero field and become equal and positive at the antiferromagnetic- 
paramagnetic phase transition (at $H_{\mathrm{C}}$ ), one of them, labeled $m_{2}$, vanishes inside the antiferromagnetic phase. For the purpose of the discussion to follow, the field corresponding to $m_{2}=0$ will be referred to as the "inversion field" $\left(H_{\text {inv }}\right)$. A line of inversion points, which is independent of $J_{\perp}^{\prime} / J_{\perp}$, can be localized on the phase diagram as shown in Fig. 2. The associated theoretical value of $\Delta / k_{B}\left(4 J_{/ / S} S^{2} / k_{B}\right)$ is $20.2 \mathrm{~K}$, which is in good agreement with the experimental value deduced from susceptibility data (16). Moreover, a reasonable estimate of the interchain coupling, $z\left|J_{\perp}\right| \approx 0.01 J_{/ /}$, is also obtained.

We now discuss the dynamics of the magnetic properties of 1. Following from our previous work (10), the dynamic measurements on powder samples have been obtained by studying the frequency dependence of the ac susceptibility, while relaxation experiments (i.e. time decay of the magnetization) have been performed on single crystals (17). The inset of Fig. 3 summarizes the results obtained from both types of measurements in zero dc-field as a function of temperature. We observe a single mode of relaxation even in the antiferromagnetic phase below $5 \mathrm{~K}$. The relaxation time of the system is thermally activated with an energy gap of $\Delta_{/} / k_{B}=54 \mathrm{~K}$. This value is fully consistent with previous analyses of other Ising-like SCM systems in the infinite chain regime, where $\Delta_{\tau}=\Delta_{A}+2 \Delta_{\S}\left(\Delta_{A}\right.$ is the anisotropy energy) (2). In fact, with $\Delta_{B} / k_{B} \approx 18 \mathrm{~K}$ measured from the $\chi T$ vs $1 / T$ data, $\Delta_{A} / k_{B}$ is estimated to be approximately $18 \mathrm{~K}$. This value is in turn very similar to those found for SCMs of the same family and consistent with susceptibility data in the transverse direction (18). This analysis shows that the dynamics of $\mathbf{1}$ at zero-field are essentially dependent on intrachain interactions, even in the antiferromagnetic phase. Similarly to the static properties (vide supra), a detailed study of the dynamics under dc-field requires single crystal measurements, which have been performed between 2.2 and $3.5 \mathrm{~K}$ using the micro-SQUID technique (13). First, the amplitude of the observed relaxation was checked and confirmed to be proportional to the susceptibility as shown in Fig. 1b. Accordingly, the data were normalized to determine the relaxation time. Typical time dependences of the magnetization plots, $m(t)$ (normalized between 0 and 1), are shown at $2.9 \mathrm{~K}$ in Fig. 3. While single exponential relaxations are measured close to zero field or when the magnetization is almost saturated, more complex shapes of 
$m(t)$ are observed close to the antiferromagnetic-paramagnetic phase transition. To obtain a simple experimental estimation of the relaxation time, $\tau$, we have taken the time when the normalized magnetization is equal to $1 / \mathrm{e}$. The deduced $\tau(H)$ curves at $2.9 \mathrm{~K}$ or $3.3 \mathrm{~K}$ are given in Fig. $4 \mathrm{a}$ or $4 \mathrm{~b}$ respectively. At $2.9 \mathrm{~K}$, and more generally below $3.2 \mathrm{~K}$, two maxima are observed in the field dependence of the relaxation time with the sharpest one near to $40 \mathrm{mT}$, i.e. close to the transition line. At higher temperature, the broad low-field maximum is nearly hidden by the sharp increase of the relaxation time close to $40 \mathrm{mT}$ (see for example at $3.3 \mathrm{~K}$ in Fig. $4 \mathrm{~b}$ ). To analyze the $\tau(H)$ curves, we still rely on a simple model based on the mean field approximation. Using this model, we obtain a set of two coupled equations, which describe the relaxation of the two normalized magnetizations. In coherence with the experiments, the linear response of the system can then be obtained. Two eigen-modes are deduced from the theory, each of them being characterized by a given relaxation time. Both modes are relevant in the antiferromagnetic phase and the slowest mode gives the theoretical magnetic relaxation time, which can be compared with the experimental results. We obtain:

$$
\frac{1}{\tau}=\frac{1}{2}\left(\frac{1-A_{1}^{\prime}}{\tau_{1}}+\frac{1-A_{2}^{\prime}}{\tau_{2}}\right)-\frac{1}{2} \sqrt{\left(\frac{1-A_{1}^{\prime}}{\tau_{1}}-\frac{1-A_{2}^{\prime}}{\tau_{2}}\right)^{2}+4 \frac{A_{1} A_{2}}{\tau_{1} \tau_{2}}}
$$

Where $\tau_{i}$ are the relaxation times for uncoupled chains, in their effective fields, $A_{i}=-\left(1-m_{i}^{2}\right)^{3 / 2} / \alpha$ and $A_{i}^{\prime}=-r\left(1-m_{i}^{2}\right)^{3 / 2} / \alpha, \quad i=1$ or $2(r$ and $\alpha$ being defined in ref. 15). From this model, slow relaxation of the magnetization is predicted even in the antiferromagnetic phase, as experimentally observed. In this ordered phase, close to the AF-paramagnetic transition line, an enhancement of the relaxation time is expected due to interchain couplings inducing a sharp maximum on the $\tau(H)$ curves, consistent with experiment (Fig. 4) (19). Deeper inside the antiferromagnetic phase, when the magnetization of the sublattice 1 is almost saturated, the dynamics are essentially due to sublattice 2 . In this regime, $A_{l}$ and $A^{\prime}{ }_{1}$ become negligible and a simplified expression of the relaxation time is obtained: $\tau \approx \tau_{2} /\left(1-A_{2}{ }^{\prime}\right)$. This expression implies the occurrence of a second maximum of the relaxation time at the inversion field. In 
this regime, the field dependence of the relaxation time is given by the dashed lines in Fig. 4. The maximum of these lines localizes the position of the inversion field. Using this approximation and the thermodynamic results which give $A_{2}{ }^{\prime}$, it has also been possible to deduce the relaxation time of an isolated chain and then finally the entire field dependence of $\tau$ can be calculated (continuous lines in Fig. 4, $\alpha=0.02$ and 0.1 were used at 2.9 and $3.3 \mathrm{~K}$ respectively as estimated from the expression of $\alpha$ for $T_{N}=5 \mathrm{~K}, r=0.3$ and $\left.4 J_{/ / S} S^{2} / k_{B}=20.2 \mathrm{~K}\right)$. The model reproduces very well the main characteristics of the experimental data in such a way that the two characteristic fields can be determined. The location of these maxima on the phase diagram shown in Fig. 2 is remarkably in agreement with (i) static measurements (maximum of susceptibility) and also (ii) the theoretical phase diagram deduced from the mean field model, considering that the corresponding fields can be identified as the inversion and transition fields respectively.

In conclusion, we have shown that the existence of an antiferromagnetic order does not prevent the observation of slow relaxation of the magnetization in a SCM-based material. Enhancement of the relaxation time is even observed close to the antiferromagnetic-paramagnetic transition line (i.e. when a small magnetic field is applied). From this point of view, by introducing large intrachain interactions between anisotropic spins one can promote high blocking temperatures in SCM-based materials independently of the presence of an ordered antiferromagnetic phase, which is a necessary consequence of even very small interchain interactions (20).

The authors thank P. Poulin and N. Hearns for critical reading of the manuscript and their useful comments. The authors are grateful to the University of Bordeaux, the CNRS, the Région Aquitaine, Sumitomo Foundation, a Grant-in-Aid for Scientific Research (Grant No. 18685007) from the Ministry of Education, Culture, Sports, Science, and Technology, Japan for funding.

1. R. J. Glauber, J. Math. Phys. 4, 294 (1963). 
2. C. Coulon, H. Miyasaka, R. Clérac, Struct. Bond. 122, 163 (2006).

3. A. Caneschi, et al., Angew. Chem. Int. Ed. 40, 1760 (2001).

4. R. Clérac et al., J. Am. Chem. Soc. 124, 12837 (2002).

5. S. K. Ritter, C\&E News 82, 29 (2004).

6. R. Sessoli, Angew. Chem. Int. Ed. 47, 5508 (2008); L. Bogani et al., J. Mater. Chem. 18, 4750 (2008).

7. P. Gambardella et al., Nature 416, 301 (2002); A. Vindigni et al., Appl. Phys. A 82, 385 (2006).

8. See for example: S. W. Choi et al., Inorg. Chem. 47, 10214 (2008); K. S. Gavrilenko et al., Chem. Eur. J. 14, 2034 (2008).

9. See Supporting Information $\mathrm{S}_{1}$ for details on synthesis and crystal structure.

10. C. Coulon et al., Phys. Rev. B 76, 214422 (2007).

11. Considering the intrachain exchange Hamiltonian: $H=-2 J_{/ /} \sum_{i} S_{i z} S_{i+1 z}$, the activation energy is $\Delta_{\xi}=4 J_{/ /} S^{2}$, where $S$ is the spin value of each magnetic unit.

12. C. Coulon et al., Phys. Rev. B 69, 132408 (2004).

13. W. Wernsdorfer, et al., J. Appl. Phys. 78, 7192 (1995); W.Wernsdorfer, N. E. Chakov and G. Christou, Phys. Rev. B 70, 132413 (2004).

14. Comparison between powder and single crystal measurements is given in Supporting Information $\mathrm{S}_{2}$.

15. The optimum was found for $r=0.3$ with $r=z^{\prime} J_{\perp}^{\prime} / z J_{\perp}, z$ and $z^{\prime}$ being the corresponding number of nearest neighbors. The same phase diagram topology is found for other positive values of $r$, 
with an AF domain located at low field $(b<1+r)$ and low temperature $(\alpha<1-r)$ with, $b=\mu H / 4 z\left|J_{\perp}\right| S^{2}$ and $\alpha=k_{B} T \exp \left(-4 J_{/ /} S^{2} / k_{B} T\right) / 4 z\left|J_{\perp}\right| S^{2}, k_{B}$ being the Boltzmann constant. Details on the theory will be given in a future publication.

16. An even better agreement with the susceptibility data is found for smaller values of $r$, although the shape of the transition line is less satisfactory for $r<0.2$ (for example $r=0.2$ gives $4 J_{/ /} S^{2} / k_{B}=$ $19.1 \mathrm{~K}$ and $r=0.1$ gives $4 J_{/ /} S^{2} / k_{B}=18.1 \mathrm{~K}$ taking $T_{N}=5 \mathrm{~K}$ ). The simplicity of the model may be at the origin of this small imperfection.

17. Powder measurements have been performed with an ac field of $0.3 \mathrm{mT}$ while single crystal measurements were collected applying an initial field $H-\delta H$ to study the relaxation towards the final field $H\left(\mu_{0} \delta H=1.4 \mathrm{mT}\right)$.

18. H. Miyasaka et al., Inorg. Chem. 42, 8203 (2003); In the present material, a $M$ vs $H$ measurement on single crystal gave $\chi_{\perp}=0.36 \mathrm{~cm}^{3} \mathrm{~mol}^{-1}$ when the field is applied perpendicular to the easy axis. This result corresponds to an anisotropy field of about $9.3 \mathrm{~T}$ and to $\Delta_{\mathrm{A}} / k_{B}=18.7 \mathrm{~K}$.

19. Note that the theory overestimates the enhancement of the relaxation time close to the transition line. This is not surprising as the mean field approximation fails in describing the critical behavior at a second order phase transition.

20. Although the relaxation time reaches its maximum close to the transition line, it has already large value in zero field. This finding is certainly important for future use of SCMs presenting larger interchain interactions. 

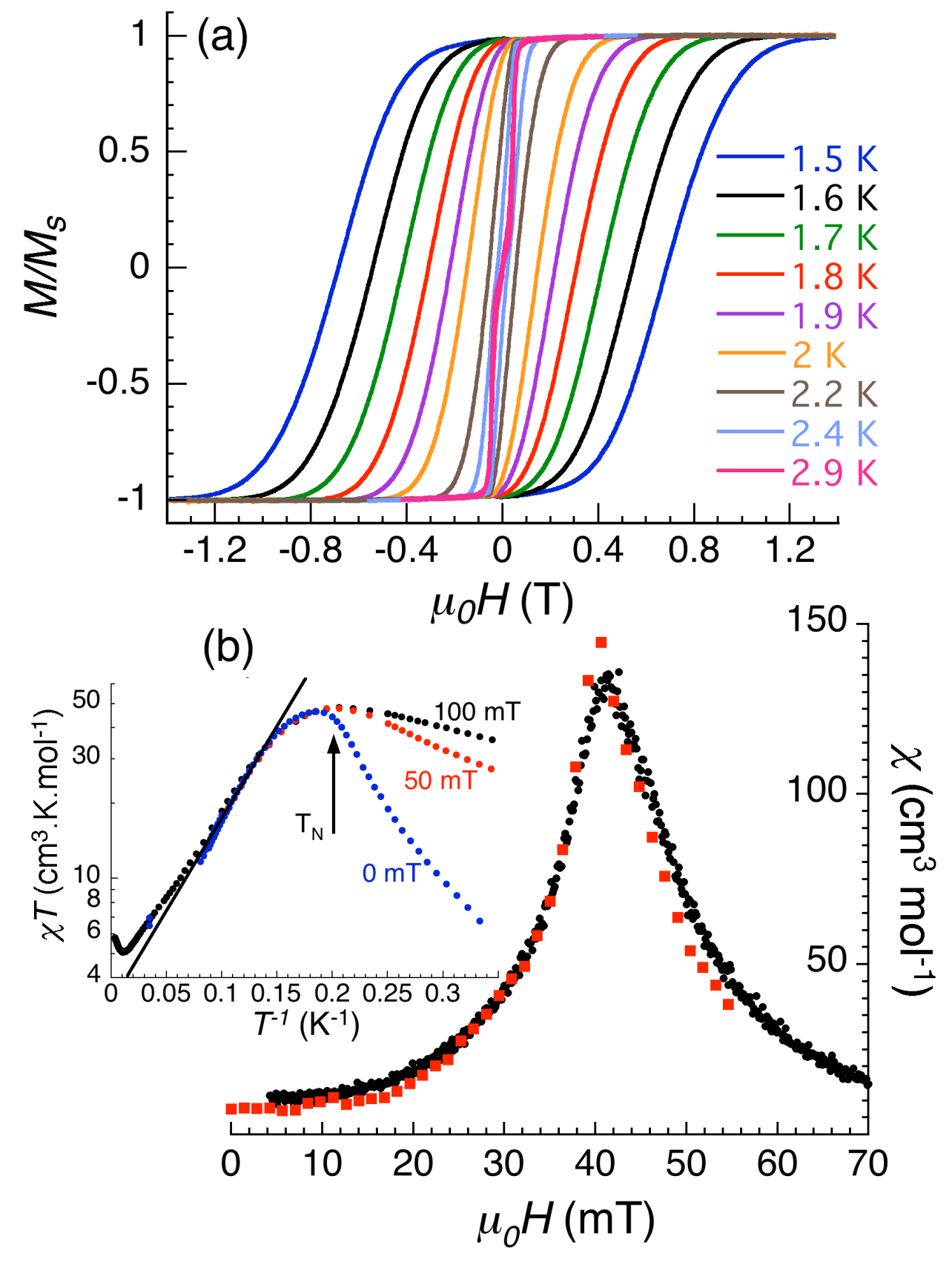

FIG. 1 (color online). (a) Field dependence of the magnetization below $2.9 \mathrm{~K}$ (field sweeping rate: 280 $\mathrm{mT} / \mathrm{s})$ measured in the easy magnetic direction $(a+c)$ for a single crystal.; (b) Field dependence of the single crystal susceptibility from dc measurements at $2.9 \mathrm{~K}$ (black dots) with a field sweeping rate of 2.2 $\mathrm{mT} / \mathrm{s}$. The susceptibility deduced from relaxation data after normalization is also shown (red squares). Powder magnetic susceptibility data at three different applied fields are shown in inset. The straight line gives the activation energy, here $\Delta_{i} / k_{B}=18 \mathrm{~K}$. 


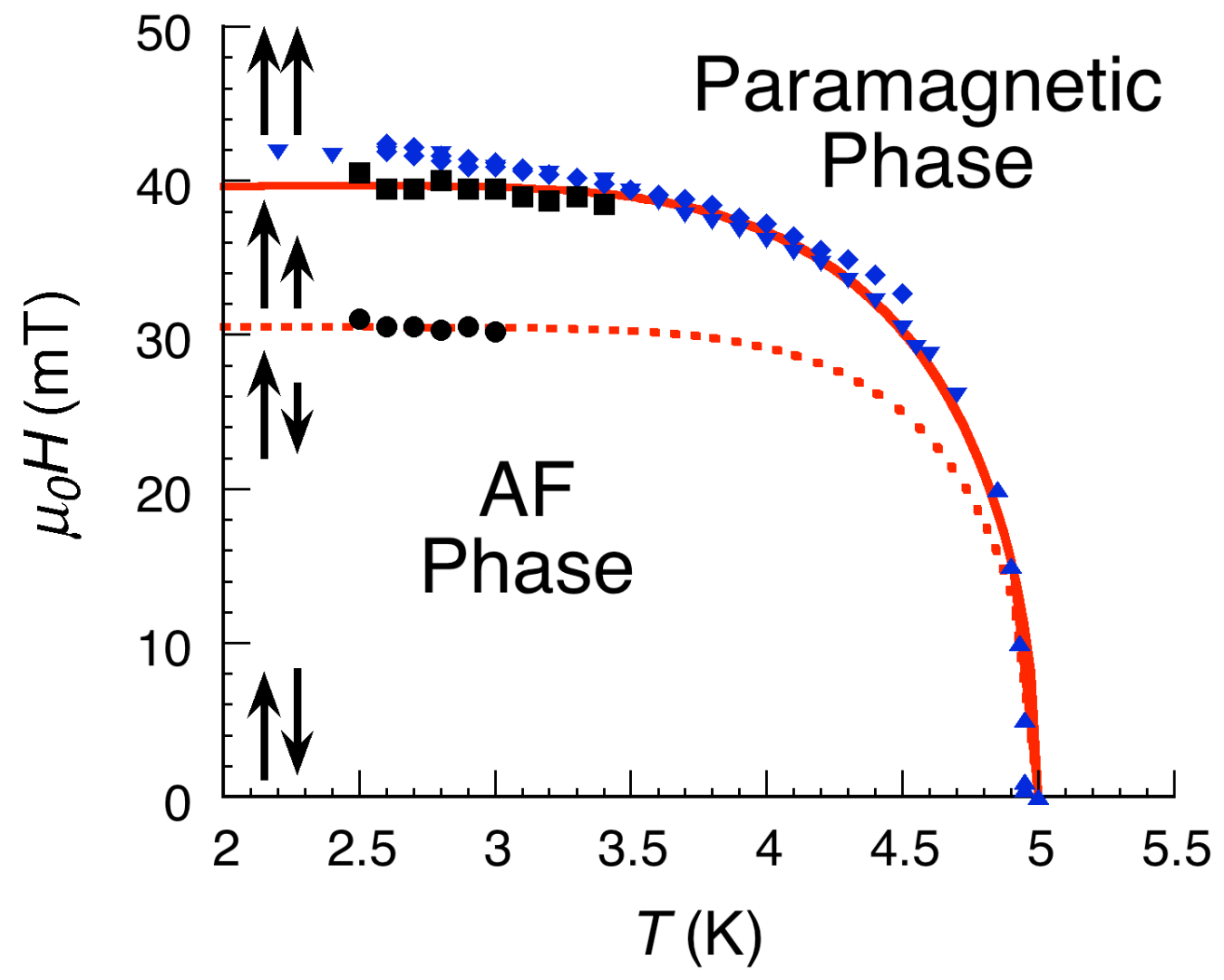

FIG. 2 (color online). Location of the maximum of susceptibility from $M(H)$ : $(\diamond)$ single crystal, $(\boldsymbol{\nabla})$ powder measurements, or from the $\mathrm{T}$ dependence of the powder ac susceptibility at a given dc field $(\boldsymbol{\Delta})$. Experimental points deduced from the dynamics measurements, ( $\mathbf{\square})$ location on the main maximum of the relaxation time and $(\mathbf{O})$ location of the second maximum (inversion point). The continuous line is the theoretical estimation of the AF - paramagnetic phase transition and the dashed line gives the line of inversion points $\left(4 J_{/} S^{2} / k_{B}=20.2 \mathrm{~K}\right.$ and $\left.r=0.3\right)$. The arrows are schematizing the orientation and magnitude of the two order parameters. 


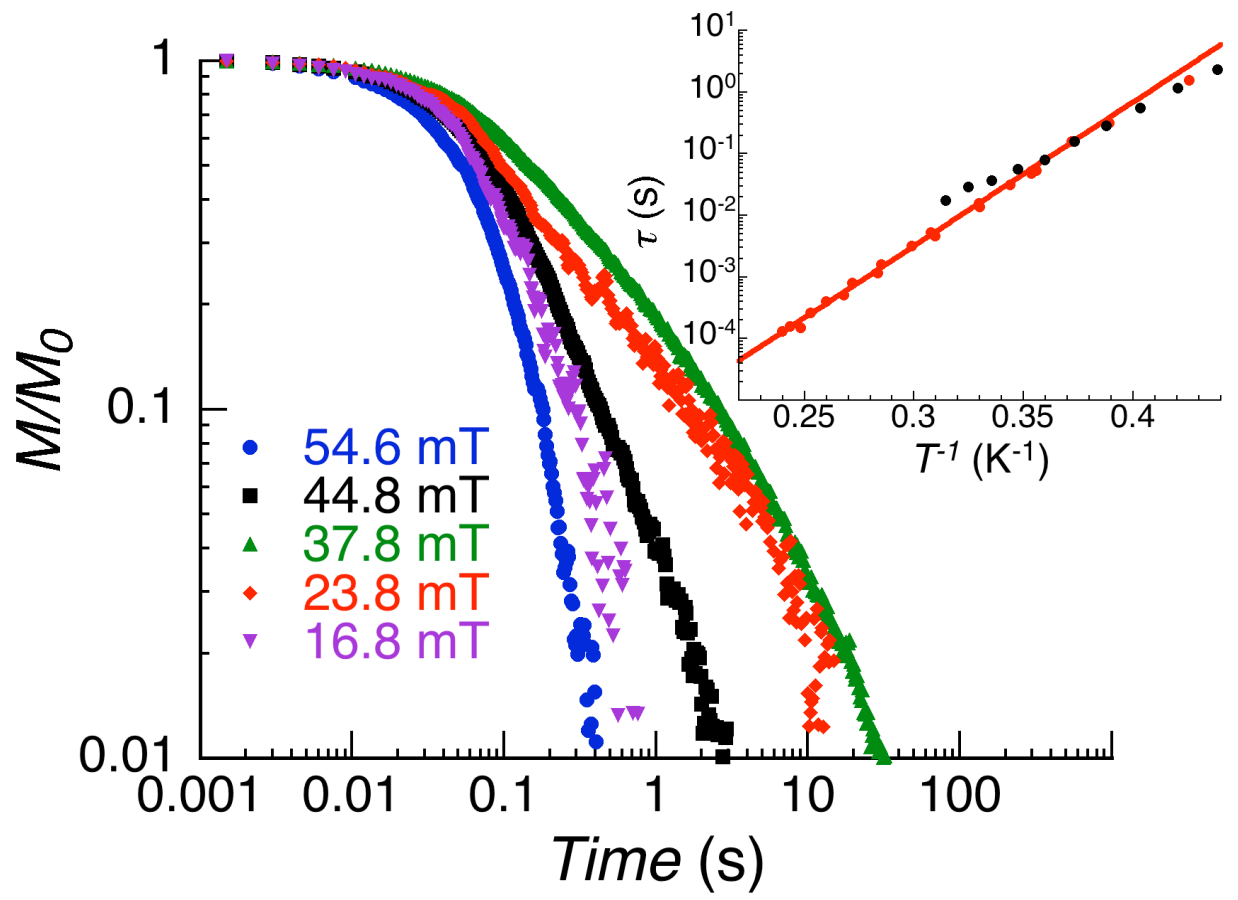

FIG. 3 (color online). Time dependence of the normalized magnetization for different values of the applied magnetic field at $2.9 \mathrm{~K}$. Inset: relaxation time versus $1 / \mathrm{T}$ in a semi-log plot in zero-dc field (data on a single crystal and powder sample are shown in black and red dots respectively). The continuous line emphasizes the activated behavior of the relaxation time with $\Delta / k_{\mathrm{B}}=54 \mathrm{~K}$ and $\tau_{0}=3.1 \times 10^{-10} \mathrm{~s}$. 


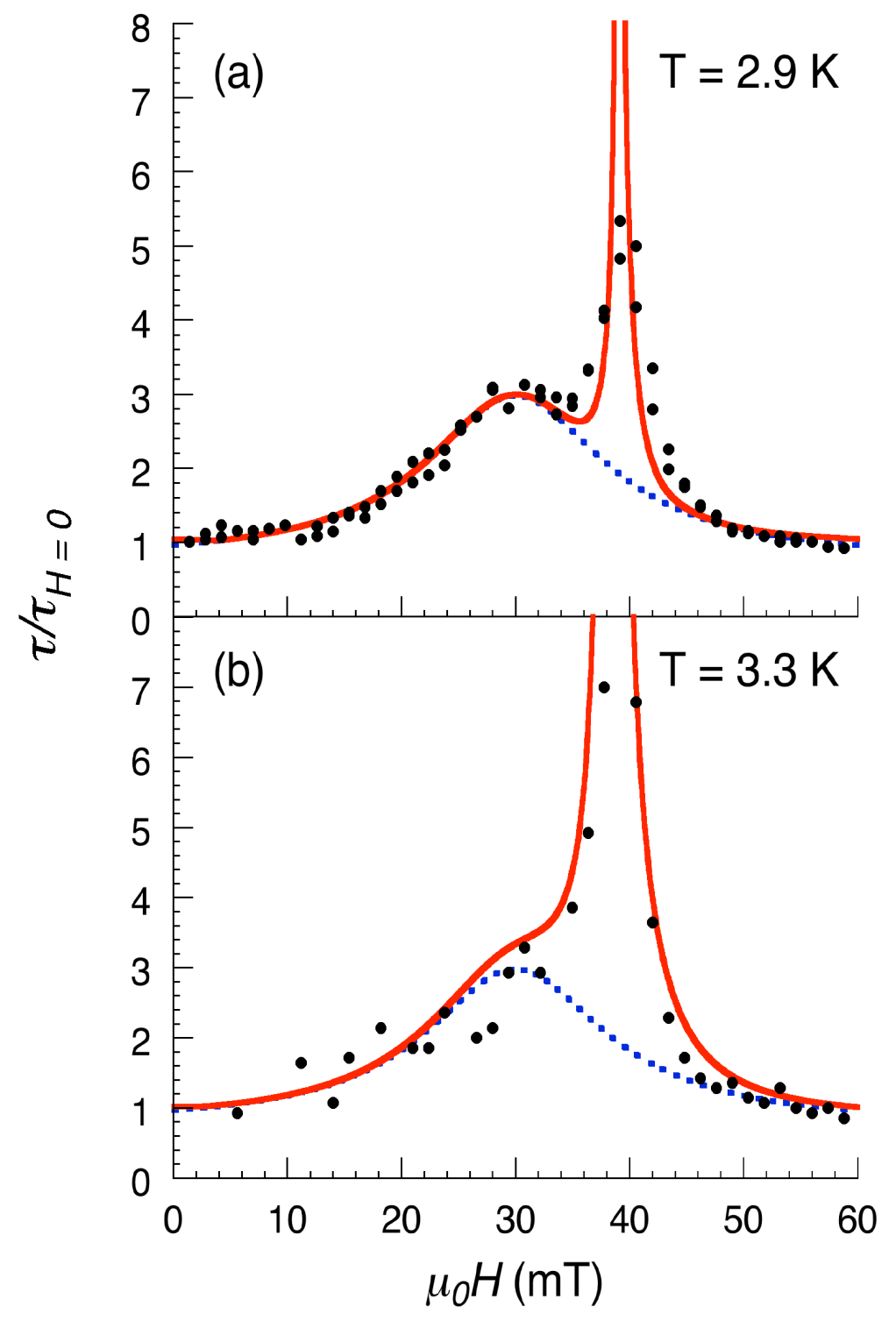

FIG. 4 (color online). Deduced relaxation time (normalized at zero field): black dots (a) at $T=2.9 \mathrm{~K}$, (b) at $T=3.3 \mathrm{~K}$. The dotted line shows the expected relaxation time when the magnetization $m_{l}$ of the sublattice \#1 is saturated. The continuous line gives the calculated relaxation time. 